\title{
Obtaining single stimulus evoked potentials with Wavelet Denoising
}

\author{
R. Quian Quiroga \\ John von Neumann Institute for Computing, \\ Forschungszentrum Jülich, \\ D - 52425 Jülich, Germany
}

October 31, 2018

PACS numbers: 5.45.Tp; 87.10.+e; 87.19.Nn

Keywords: wavelet, denoising, EEG, evoked potential.

corresponding author: tel/fax: +49 2461 612302/2430

e-mail: R.QuianQuiroga@fz-juelich.de 


\begin{abstract}
We present a method for the analysis of electroencephalograms (EEG). In particular, small signals due to stimulation, so called evoked potentials, have to be detected in the background EEG. This is achieved by using a denoising implementation based on the wavelet decomposition.

One recording of visual evoked potentials, and recordings of auditory evoked potentials from 4 subjects corresponding to different age groups are analyzed. We find higher variability in older individuals. Moreover, since the evoked potentials are identified at the single stimulus level (without need of ensemble averaging), this will allow the calculation of better resolved averages. Since the method is parameter free (i.e. it does not need to be adapted to the particular characteristics of each recording), implementations in clinical settings are imaginable.
\end{abstract}




\section{Introduction}

Evoked potentials (EP) are the alterations of the ongoing electroencephalogram (EEG) due to stimulation (e.g. tone, light flash, etc). They are time locked to the stimulus and they have a characteristic pattern of response that is more or less reproducible under similar experimental conditions [2, 17]. In order to study the response of the brain to different tasks, sequences of stimuli can be arranged according to well defined paradigms. This allows the study of different sensitive or cognitive functions, states, pathologies, etc, thus making the EPs an invaluable tool in neurophysiology.

Due to the low amplitudes of evoked potentials in comparison with the ongoing EEG, they are hardly seen in the original EEG signal, and therefore several trials (i.e. data segments including the pre- and post-stimulus activity) are averaged in order to enhance the evoked responses. Since EPs are time locked to the stimulus, their contribution will add while the ongoing EEG will cancel (see [15] for a quantitative study of time locking and amplitude increases of the EPs in comparison with the spontaneous EEG). However, when averaging, information related with variations between the single trials is lost. This information could be relevant in order to study behavioral and functional processes, habituation, refractoriness, and it could also help to identify pathologies when the information from the average evoked potential is not clear. Moreover, in many cases a compromise must be made when deciding on the number of trials in an experiment. If we take a large number of trials we optimize the EP/EEG ratio but if the number of trial is too large, then we could deal with effects such as tiredness, which eventually corrupts the average results. This problem can be partially solved by tak-

ing sub-ensemble averages (i.e. consecutive averages of a few single sweeps). 
However, in many cases the success of such procedure is limited, especially when not many trials can be obtained or when characteristics of the evoked potentials change from trial to trial.

Several methods have been proposed in order to filter averaged EPs (see [12]). The success of such methods would imply the need of less number of trials and would eventually allow the extraction of single trial evoked potentials from the background EEG. Although averaging has been used since the middle 50's, up to now none of these attempts has been successful in obtaining single trial EPs, at least in a level that they could be applied to different type of EPs and that they could be implemented in clinical settings. Most of these approaches involves Wiener filtering [19, 10] (or a "minimum mean square error filter" based on auto and cross-correlations in [14]) and have the common drawback of considering the signal as a stationary process. Since EPs are transient responses related with specific time and frequency locations, such time-invariant approaches are not likely to give optimal results. For this reason, de Weerd and coworkers [7, 8] introduced a time-varying generalization for filtering averaged evoked potentials. The time-variant Wiener filter they proposed is clearly more suitable for the analysis of evoked potentials but with the caveats that such filter bank implementation does not give a perfect reconstruction, and that it is based on the Fourier Transform (therefore the signal being decomposed in bases of sines and cosines with the drawbacks that this imposes, as we will describe later).

These limitations, as well as the ones related with time-invariant methods can be solved by using the wavelet formalism. The Wavelet Transform is a time-frequency representation proposed first in [11], that has an optimal resolution both in the time and frequency domains and has been successfully applied to the study of EEG-EP signals [16, 18, 6, 匇. The objective of the 
present study is to follow an idea originally proposed in [1], and to present a very straightforward method based on the Wavelet Transform to obtain the evoked responses at the single trial level. The key point in the denoising of evoked potentials is how to select in the wavelet domain the activity representing the signal (the EPs) and then eliminate the one related with noise (the background EEG). In fact, the main difference between our implementation and previous related approaches is in the way that the wavelet coefficients are selected. Briefly, such choice should consider latency variations between the single trial responses and it should not introduce spurious effects in the time range where the EPs are expected to occur. In this respect, the denoising implementation we propose will allow the study of variability between single trials, an information that could have high physiological relevance.

The paper is organized as follows: In section $⿴$ the data sets to be analyzed are described. Section 3 gives the mathematical background of the Wavelet Transform and the multiresolution decomposition. The implementation of the denoising of EPs is presented in section 6 , together with a discussion of the advantages of the present implementation in comparison with previous approaches. The application of the method to visual and auditory EPs is shown in sections 5 and 6 , respectively. Finally, in section 7 the conclusions are drawn. 


\section{Details of the data}

We will study evoked potential recordings obtained with an oddball paradigm upon two different stimulus modalities: visual evoked potentials (VEP) and auditory evoked potentials (AEP). The robustness of the method is stressed by the fact that although an (almost) identical implementation was used for both data sets, these were taken in different laboratories and under different recording settings.

\subsection{Visual evoked potentials}

Visual evoked potentials from one normal subject were obtained by using a checkerboard light pattern. Two different visual stimuli were presented in a pseudo-random order (oddball paradigm): $75 \%$ of the stimuli were the so called "non-target" (a color reversal of the checks) and the other 25\% were the deviant stimuli or "target" (also a color reversal but with a half check displacement of the pattern). The subject was instructed to ignore the nontarget stimuli and to count the number of appearances of the target ones (see [16] for more details on the experimental setup). Scalp recordings were obtained from the left occipital (O1) electrode (near to the location of the visual primary sensory area) with linked earlobes reference. Sampling rate was $250 \mathrm{~Hz}$ and after bandpass filtering in the range $0.1-70 \mathrm{~Hz}, \sim 2 \mathrm{sec}$ of data (256 data pre- and 256 data post-stimulation) were saved on a hard disk. Inter-stimulus intervals varied randomly between $2.5-3.5$ sec. The recording session consisted of 200 stimuli presentations. From a total of 50 target stimuli, after rejection of trials with artifacts (contamination of the recording with spurious activity; e.g. blinking) 30 were selected for further analysis. 
Two evoked responses can be observed with this paradigm (see average response on the uppermost left plot in figure 2): First, a sensory related positive peak at about $100 \mathrm{~ms}$ after stimulation (P100) followed by a negative rebound (N200), that appear both upon non-target and target stimuli. Second, a positive peak at about 400-500 ms after stimulation (P300) appearing only upon target stimuli and related with the cognitive process of recognizing these stimuli as deviant.

\section{$2.2 \quad$ Auditory evoked potentials}

Evoked potentials of two young (37 and 45 years) and two old (70 and 74 years) normal subjects were obtained in an eyes open condition from a scalp central electrode $(\mathrm{Cz})$ with linked earlobes reference. As in the case of VEP an oddball paradigm was used, the non-target stimuli being tones of 1000 $\mathrm{Hz}$ and the target ones being tones of $500 \mathrm{~Hz}$. The inter-stimulus interval was 2 sec. In each session, 100 stimuli (75 non-target and 25 target) were presented and after artifact rejection between $40-50$ non-target and between 11 - 16 target stimuli were selected for further analysis. For each trial, after digital filtering in the range $1-70 \mathrm{~Hz}, 1 \mathrm{sec}$ of data was saved on a hard disc (from $0.2 \mathrm{sec}$ pre- to $0.8 \mathrm{sec}$ post-stimulation). Sampling rate was $204.8 \mathrm{~Hz}$ and the data of each trial was extended with symmetric border conditions in order to have a number of 256 data points per trial.

As with VEP, in the case of AEPs two main responses appear: the N100P200 peaks (polarity is reversed in comparison to VEP) and a P300 response (but appearing earlier than in the VEP case, at about 300ms after stimulation). 


\section{Wavelet Transform}

\subsection{From Fourier to Wavelets}

The Fourier Transform of a given signal is defined as its inner product with complex exponential (sines and cosines) functions of different frequencies. It allows a better visualization of the periodicities of the signal, especially when several frequencies are superposed. However, Fourier Transform gives no information about the time location of these periodicities and it further requires stationarity of the signal.

By tapering ("windowing") the complex exponential mother functions of the Fourier Transform, the Short Time Fourier Transform (STFT) gives a time evolution of the frequencies that can be obtained just by sliding the windows throughout the signal. The STFT gives an optimal time-frequency representation, but a critical limitation appears when windowing the data due to the uncertainty principle (see [4]). If the window is too narrow, the frequency resolution will be poor, and if the window is too wide, the time localization will be not so precise. Data involving slow processes will require wide windows while a narrow window will be more suitable for data with fast transients (high frequency components). Then, owing to its fixed window size, the STFT is not suitable for analyzing signals involving different range of frequencies, as in the case of EPs.

Grossmann and Morlet [1] introduced the Wavelet Transform in order to overcome this problem. The main advantage of wavelets is that they have a varying window size, being wide for low frequencies and narrow for the high ones, thus leading to an optimal time-frequency resolution in all the frequency ranges [4]. Furthermore, owing to the fact that windows are adapted to the transients of each scale, wavelets do not need stationarity. 
The Continuous Wavelet Transform (CWT) of a signal $x(t) \in L^{2}(\mathcal{R})$ is defined as the inner product between the signal and the wavelet functions $\psi_{a, b}(t)$

$$
W_{\psi} x(a, b) \equiv C_{a, b}=\left\langle x(t), \psi_{a, b}(t)\right\rangle
$$

where $C_{a, b}$ are the wavelet coefficients and $\psi_{a, b}(t)$ are dilated (contracted) and shifted versions of a unique wavelet function $\psi(t)$

$$
\psi_{a, b}(t)=|a|^{-1 / 2} \psi\left(\frac{t-b}{a}\right)
$$

( $a, b$ are the scale and translation parameters, respectively). The CWT gives a decomposition of $x(t)$ in different scales, tending to be maximum at those scales and time locations where the wavelet best resembles $x(t)$. Contracted versions of $\psi_{a, b}(t)$ will match high frequency components of $x(t)$ and on the other hand, dilated versions will match low frequency oscillations.

The CWT maps a signal of one independent variable $t$ onto a function of two independent variables $a, b$. This procedure is redundant and not efficient for algorithm implementations. In consequence, it is more practical to define the Wavelet Transform only at discrete scales $a$ and discrete times $b$ by choosing the set of parameters $\left\{a_{j}=2^{j}, b_{j, k}=2^{j} k\right\}$, with $j, k \in \mathcal{Z}$. We obtain then the discrete wavelet family

$$
\psi_{j, k}(t)=2^{-j / 2} \psi\left(2^{-j} t-k\right) \quad j, k \in \mathcal{Z},
$$

that under appropriate conditions [ [4] forms a basis of $L^{2}(\mathcal{R})$, each wavelet function having a good localization in the time and frequency domains. In analogy with eq.(1) we define the Dyadic Wavelet Transform as 


$$
W_{\psi} x(j, k) \equiv C_{j, k}=\left\langle x(t), \psi_{j, k}(t)\right\rangle
$$

For well defined wavelets it can be inverted, thus giving the reconstruction of $x(t)$

$$
x(t)=\sum_{j, k} C_{j, k} \hat{\psi}_{j, k}(t) \quad j, k \in \mathcal{Z}
$$

where $\hat{\psi}$ is the dual of $\psi$ (in case of orthogonal wavelets, $\hat{\psi}$ and $\psi$ are identical).

\section{$3.2 \quad$ B-Splines wavelets}

Another advantage of the Wavelet Transform over Fourier based methods is that the functions to be matched with the signal are not necessarily sinusoidal ones (or modulated sinusoidal in the case of the STFT). In fact, there are many different functions suitable as wavelets, each one having different characteristics that are more or less appropriate depending on the application. Irrespective of the mathematical properties of the wavelet to choose, a basic requirement is that it looks similar to the patterns we want to localize in the signal. This allows a good localization of the structures of interest in the wavelet domain and moreover, it minimizes spurious effects in the reconstruction of the signal via the inverse Wavelet Transform (5).

For this study, we choose quadratic biorthogonal B-Splines [5] as mother functions (see Fig.11) due to their similarity with the evoked responses. BSplines are piecewise polynomials that form a base in $L^{2}$ [4, 21]. We remark the following properties that make them optimal in signal analysis (see [21, 4, 5] for details): they are (anti-) symmetric, smooth, they have a nearly optimal time-frequency resolution and they have compact support. 


\subsection{Multiresolution decomposition}

The information given by the dyadic Wavelet Transform can be organized according to a hierarchical scheme called multiresolution analysis [13]. If we denote by $W_{j}$ the subspaces of $L^{2}$ generated by the wavelets $\psi_{j, k}$ for each level $j$, the space $L^{2}$ can be decomposed as a direct sum of the subspaces $W_{j}$,

$$
L^{2}=\sum_{j \in \mathcal{Z}} W_{j}
$$

Let us define the subspaces

$$
V_{j}=W_{j+1} \oplus W_{j+2} \oplus \ldots \quad j \in \mathcal{Z}
$$

The subspaces $V_{j}$ are a multiresolution approximation of $L^{2}$ and they are generated by scalings and translations of a single function $\phi_{j, k}=\phi\left(2^{-j} n-k\right)$ called the scaling function (see proof in [13]). Then, for the subspaces $V_{j}$ we have the complementary subspaces $W_{j}$, namely:

$$
V_{j-1}=V_{j} \oplus W_{j} \quad j \in \mathcal{Z}
$$

Let us suppose we have a discretely sampled signal $x(n) \equiv a_{0}(n)$ with finite energy. We can successively decompose it with the following recursive scheme

$$
a_{j-1}(n)=a_{j}(n)+d_{j}(n)
$$

where the terms $a_{j}(n)=\sum_{k} a_{j-1}(k) \phi_{j, k}(n) \in V_{j}$ give the coarser representation of the signal and $d_{j}(n)=\sum_{k} a_{j-1} \psi_{j, k}(n) \in W_{j}$ give the details for 
each scale $j=0,1, \cdots, N$. For any resolution level $N>0$, we can write the decomposition of the signal as

$$
x(n) \equiv a_{0}(n)=d_{1}(n)+d_{2}(n)+\ldots+d_{N}(n)+a_{N}(n)
$$

This method gives a decomposition of the signal that can be implemented with very efficient algorithms due to the recursiveness of the decomposition (eq.(9) ). Moreover, Mallat [13 showed that each detail $\left(d_{j}\right)$ and approximation signal $\left(a_{j}\right)$ can be obtained from the previous approximation $a_{j-1}$ via a convolution with (FIR or truncated IIR) high-pass and low-pass filters, respectively.

De Weerd and coworkers [7, 8] filtered averaged evoked potentials by using octave filter banks with the bandwidth of each filter being proportional to their center frequency. This approach is in principle similar to the one obtained with wavelets but it has some disadvantages. First, it repeatedly makes use of the Fourier Transform, therefore being forced to decompose the signal in bases of sinus and cosinus. On the contrary, with wavelets we have the advantage of choosing the mother wavelet from a collection of available functions with properties that will be more or less suitable depending on the application (see previous section). Second, as already remarked by Bertrand et al [3] the filter bank implementation of de Weerd does not allow a perfect reconstruction of the signal \&

The gray curves in Fig.2 show the decomposition of an averaged (over 30

1 The computing time $(\mathcal{O}(M), M$ : number of data points) is even faster than the one of the fast Fourier Transform $(\mathcal{O}(M \cdot \log M))$.

2 However we should remark that the multiresolution decomposition gives a perfect reconstruction (disregarding border effects) because the mother functions $\psi_{j, k}(t)$ of eq. (3) form a basis of $L^{2}(\mathcal{R})$. This is not to be confounded with the requirement of orthogonality as claimed in 3 . 
trials) visual evoked potential. In this and all other cases studied we used a 5 levels decomposition, thus having 5 scales of details $\left(d_{1}\right.$ to $\left.d_{5}\right)$ and a final approximation $\left(a_{5}\right)$. On the left side we plot the wavelet coefficients and on the right side the actual components/decomposition. The sum of all the reconstructions gives again the original signal (gray curve of the uppermost right plot). The lower levels give the details corresponding to the high frequency components and the higher levels the ones correspond to the low frequencies. 


\section{Denoising of evoked potentials}

\subsection{Implementation of the method}

As proposed by Donoho [9], the conventional definition of denoising implies a thresholding criterion in the wavelet domain. The signal is recovered from noisy data just by setting to zero those wavelet coefficients below a certain threshold (hard denoising) or with the use of a smoother transformation (soft denoising). However, this procedure is not optimal for recovering the evoked potentials because these ones are of the order or even smaller than the background EEG. Therefore, instead of using a thresholding criterion, we implemented a denoising based on the specific time and frequency localizations of the evoked responses.

Note in Fig.2 that the P100-N200 response is correlated mostly with the first post-stimulus coefficients in the details $d_{4}-d_{5}$ and the P300 is mainly correlated with the coefficients at about $400-500 \mathrm{~ms}$ in $a_{5}$. In consequence, a straightforward way to avoid the fluctuations related with the ongoing EEG and get only the peaks of interest, is just by equaling to zero those coefficients not correlated with the EPs.

The black traces in the left side of fig.2 show the coefficients kept for the reconstruction of the P100-N200 and P300 responses and the black curves on the right side show the contributions of each level obtained by eliminating all the other coefficients. Note that in the final reconstruction of the averaged response (black curve in the uppermost right plot) background EEG oscillations are cancelled. We should remark that this is usually difficult to achieve with a Fourier filtering approach (especially in averages of less number of trials) due to the different time and frequency localizations of the P100 and P300 responses, and overlapping frequency components of these peaks and 
the ongoing EEG. In this context, the main advantage of Wavelet denoising over conventional filtering is that we can select different "time windows" for the different scales. Advantages of wavelets over Fourier for simultaneously filtering early and middle latency (up to $50 \mathrm{~ms}$ after stimulation) averaged auditory EPs were described by Bertrand et al. [3]. Moreover, these authors proposed a "wavelet based" 3 post-averaging Wiener filtering for cleaning averaged evoked potentials.

Since as we mentioned in the introduction, evoked potentials are activity time locked to the stimulation, we could use this feature to reconstruct the contribution of the single trials to the averaged EPs. This will allow the visualization of the EPs at the single trial level as we will show in the next section.

Let us remark a critical point when implementing the denoising of the EPs. This is the choice of which coefficients to keep and which to eliminate. On one hand, choosing a wide range of scales ("frequency window") allows a better reconstruction of the morphology of the EPs (again we remark that the selection of an appropriate wavelet function plays an important role in this respect). Also, choosing a wide "time window" of coefficients makes the method more sensitive to latency differences (jitters) between trials (in the extreme case of keeping one single coefficient, the denoised signal will be just the wavelet function with its amplitude proportional to the coefficient). On the other hand, if we choose a wide ("conservative") range of coefficients

\footnotetext{
${ }^{3}$ Instead of the usual Wavelet Transform, Bertrand and coworkers used an analogous transform (the wavelet functions being not anymore dilated and translated versions of a unique wavelet) in order to avoid border problems in the reconstruction. However, such correction seems to be unnecessary in general because border problems can be easily avoided just by having enough pre- and post-stimulus data as is usually the case in EP recordings.
} 
we would not enough eliminate the background EEG activity in order to recognize the EPs (in the extreme case of keeping all coefficients we will just reconstruct the original signal). In this respect we propose to use test signals, such as an spontaneous EEG (or a pre-stimulus EEG segment as we will show in fig.5) in order to check for eventual spurious interpretations due to an unfortunate selection of the coefficients.

We heuristically found the selection of those coefficients remarked in black traces in Fig.2 an optimal compromise between EP resolution and sensitivity to variations between trials. As we will show in the next sections, they allow the visualization of the single trial EPs and also they cover a reasonable time and scale ranges where the EPs are physiologically expected to occur.

We should also mention that the wavelet coefficients to be kept could be smoothed by using, e.g. soft thresholding [9] in order to decrease border effects. Although the hard thresholding we used can introduce spurious border fluctuations (see for example in Fig.2 the positive deflection in the denoised signal between -0.2 and 0 sec), these are outside the time range of physiological interest of the evoked responses.

In summary the method consist in the following steps:

1. The averaged EP is decomposed by using the wavelet multiresolution decomposition.

2. The wavelet coefficients not correlated with the average evoked potential (but also considering a time range in which single trial EPs are expected to occur) are identified and set to zero.

3. The inverse transform is applied, thus obtaining a denoised signal.

4. The denoising transform defined by the previous steps is applied to the single trials. 
5. Finally, validity of the results can be checked by applying the method to EEG test signals.

We remark that for all the calculations done in this study we keep the same set of coefficients (with the slight exception that for auditory evoked potentials we do not keep the last coefficient of level $A_{5}$ because auditory P300 responses appear earlier than in the visual case). In other words, once the coefficients are chosen (steps 1-3), the method is parameter free and does not need to be adjusted for different EEG/EP ratios, latency variability, number of trials or other differences between subjects, electrodes, etc.

\subsection{Comparison with previous works}

The criteria for choosing which wavelet coefficients are correlated with the signal and which ones with noise is the key feature of the different denoising implementations. This is in fact our main difference with previous approaches. As already mentioned, Donoho [9] proposed a thresholding criteria, something not suitable for separating evoked potentials from the background EEG activity. The aim of those methods using time-variant [19, 10], timeinvariant [7, 8] and wavelet based [3] Wiener filtering was to clean averaged EPs rather than to obtain single trial EPsf. Therefore, they did not consider latency variations between trials, one of the most important features obtained from single trial analysis in comparison with ensemble averaging. A similar remark is applicable to the work of Demiralp et al [6]. These authors correlated one single wavelet coefficient with the P300 response, then using its sign for discriminating between trials with and without P300. By

\footnotetext{
${ }^{4}$ However, since average filtering implies the need of less number of trials, the idea of single trial reconstruction is implicitly involved.
} 
selecting trials in this way, they succeeded in obtaining better averages of the P300.

Mc Gillen and Aunon [14] and Bartnik et al [1], instead, aimed at obtaining single trial evoked potentials. The first authors proposed a filter based in auto- and cross-correlations that however, due to its time-invariance, is not optimal for the analysis of EPs. This is the main explanation for the spurious differences between the original and the filtered signals observed in their examples (figs. 3 and 4 in ref.[14]). Bartnik and coworkers introduced a denoising implementation similar to the one presented by us. However, based on correlation and discriminant analysis (using as input the wavelet coefficients from the average and the single trials) they proposed an automatic criterion for finding which wavelet coefficients best distinguishes the EP from the EEG. In comparison with our approach, this criterion has the the following drawbacks: First, it is not appropriate if more than one peak is present, as e.g. in the case of the P100-N200 and P300 responses to be shown in the next section. Second, it is in principle not sensitive to variations between trials (i.e. variations in latency will be cancelled in the average and consequently, they will be disregarded by the correlation analysis). Third, a denoising based in such correlation criterion is likely to alter the morphology of the EPs. In particular, in the example shown by Bartnik et al the denoised signals look much smoother than the original ones, in many cases worsening the recognition and time localization of the EPs (see fig.7 in ref.[1]). 


\section{Application to Visual Evoked Potentials}

Figure 3 shows the first 15 single trials corresponding to the average of Fig.2. Note that with denoising (black curves) we can distinguish the evoked responses P100-N200 and P300 in most of the trials. Note also that these responses are not easily identified in the original signal due to their similarity with the ongoing EEG. We already can observe some variability between the trials, e.g. in the trials \#2,\#13 the EPs are practically not present. For easier visualization, in Fig. (1) we plot the single trial evoked potentials with and without denoising (left and right side plot, respectively) by using contour plots. In the denoised plot we observe between 100-200ms a yellow/red pattern followed by a blue one corresponding to the P100-N200 peaks. These responses remain more or less stable during the whole recording session. The more unstable and wider yellow/red pattern at about 400-600ms correspond to the cognitive related P300 responses. Noteworthy, all these patterns are more difficult to recognize in the original signal (left plot).

In order to check that the better visualization obtained after denoising is not just an artifact of the method, in Fig.5 we show the results of the same analysis applied to an spontaneous EEG signal (without EPs). As the EEG signal, we took for each trial of the VEP recording set the data segments corresponding to the second previous to stimulation (we assume we can disregard pre-stimulus effects, such as expectation). In contrast to the previous figure we only observe some randomly appearing spots that do not form a coherent pattern as when the EPs are present. 


\section{Application to Auditory Evoked Potentials}

Auditory target and non-target evoked potentials of a typical subject (427) are shown in Figs.6 and 7, respectively. In both figures we observe a blue pattern at about $100 \mathrm{~ms}$ after stimulation corresponding to the N100, followed by a yellow/red pattern corresponding to the P200. Upon target stimuli we can also identify a yellow pattern at about 300ms corresponding to the P300 responses. The P300 is better defined in the first 5 trials, later it appears more spread and unstable.

We further study the variability of the N100 peaks upon non-target stimuli (variability of P300 upon target stimuli can be studied in a similar way). We first obtained from the denoised responses the amplitude and latency of the N100 peaks, which where localized as the maximum in a time window between $50-180 \mathrm{~ms}$ after stimulation. We remark that this is hard to implement in the original signal due to the presence of the ongoing EEG. We then calculated the mean and standard deviation of the peak amplitude and latency. The first three columns of Table 1 show the results for the four subjects studied and the control EEG signal (without EPs) already described in Fig.5. The two different groups of subjects (young and old) can not be distin-

guished from the mean amplitudes or latencies (with exception of the fourth subject, which will be discussed later). However, we observe a higher variability both in amplitude and latency for the older subjects. These variations are even larger for the control EEG signal. We remark that our present goal is to illustrate how we can obtain information not seen in the EP averages rather than making any physiological conclusion. In this context, a detailed quantitative analysis of the variability between single trials will be reported elsewhere with a larger database of subjects and statistical validation. 
We also calculated the cross-correlations between the single trials and the average for each subject, thus having a measure of "how good" the single trials resemble the average EP. Then we computed selective averages using only those trials whose cross-correlation with the average was larger than 0.4 ๒. Although we will show selective averages for the original and denoised signals, cross-correlations were only computed with the denoised ones in order to avoid effects of random correlations of the background EEG. As implemented in 20 for pre-filtered trials, for the selected trials we calculated lag corrected averages, i.e. by shifting each trial $\Delta_{i}=t_{a v}-t_{i}$ data points, where $t_{a v}$ is the latency of the average and $t_{i}$ the one of each single sweep. The last two columns of table 1 reports the mean cross-correlation values and the number of trials selected for each subject. As expected, the EEG signal has a lower mean cross-correlation than the EPs.

In Fig.8 we show the averages, the selective averages and the shift corrected ones for the 4 subjects studied and the control EEG. On the left plots the averages with the original signals are shown and on the right plots the ones with the denoised ones. We first observe that in the denoised signals the spontaneous EEG oscillations are cancelled, thus having a clearer visualization of the EPs. For the first three subjects, in both original and denoised signals the selective averages show a better definition of the EPs. The same holds for the shifted averages, especially in the third case due to its larger latency variation (see Table 1). In the forth subject as in the case of the EEG, neither the selective and the shifted averages show a better identification of the EPs in comparison with the background activity. Note also that this is expected from their low cross-correlation values. Again, a more reliable dis-

\footnotetext{
${ }^{5}$ Similarly, a cross-correlation criteria can be also implemented for separating different type of evoked responses as shown in [20].
} 
tinction between subjects with a very large EP variability and control EEGs would require the statistical analysis of a larger sample, something that it is beyond the scope of the present study. 


\section{Conclusions}

We presented a method for extracting evoked potentials from the background EEG. It is not limited to the study of EPs/EEGs and similar implementations can be used for recognizing transients even in signals with low signal to noise ratio. The denoising of evoked potentials allowed the study of the variability between the single responses, information that could have a high physiological relevance in the study of different brain functions, states or pathologies. It could be also used to eliminate artifacts that do not appear in the same time and frequency ranges of the relevant evoked responses. In passing, we showed that the method gives better averaged evoked potentials due to the high timefrequency resolution of the Wavelet Transform, this being hard to achieve with conventional Fourier filters. Moreover, since trials with "good" evoked responses can be easily identified, it was possible to do selective averages or even jitter corrected ones, with a resulting better definition of the averaged EPs. These advantages could significantly reduce the minimum number of trials necessary in a recording session, something of high importance for avoiding behavioral changes during the recording (e.g. effects of tiredness) or, even more interesting, for obtaining EPs under strongly varying conditions, as with children, or patients with attentional problems.

The idea of denoising for obtaining single trial evoked potentials together with a single example were already presented in [1]. The main difference with these authors is in the criteria for selecting the wavelet coefficients. This is crucial for obtaining an optimal implementation that is physiologically plausible (e.g. allowing variations between trials) and that minimizes the presence of spurious effects in the time range of the evoked responses.

Finally, We should remark that once the wavelet coefficients to be used 
for denoising were selected (according to the evoked responses to focus on), the procedure for obtaining single trial EPs and for improving the averaged responses (due to denoising of the averages, to selective averaging or to jitter corrections) was done fully automatically for all trials and patients. Therefore, implementations of the method as a complementary information to routine EEG/EP analysis are imaginable.

\section{Acknowledgments}

We are very thankful to Dr. H. García for the AEP data and to Prof. E. Başar and Dr. M. Schürmann for the VEP data. We are also very thankful to Prof. P. Grassberger for stimulating discussions and a careful reading of this manuscript. 


\section{References}

[1] Bartnik EA, Blinowska KJ and Durka PJ. Single evoked potential reconstruction by means of wavelet transform. Biol. Cybern., 1992; 67: $175-181$

[2] Başar E. EEG-Brain dynamics. Relation between EEG and brain evoked potentials. Amsterdam: Elsevier, 1980.

[3] Bertrand O, Bohorquez J and Pernier J. Time-frequency digital filtering based on an invertible wavelet transform: an application to evoked potentials. IEEE Trans. Biomed. Eng., 1994; 41: 77-88.

[4] Chui C. An introduction to wavelets. Academic Press, San Diego, 1992.

[5] Cohen A,Daubechies I and Feauveau JC. Bi-orthogonal bases of compactly supported wavelets. Comm. Pure Appl. Math, 1992; 45: 485-560.

[6] Demiralp T, Ademoglu A, Schürmann M, Başar-Eroglu C and Başar E. Detection of P300 waves in single trials by the wavelet transform. Brain and language, 1999; 66: 108-128.

[7] de Weerd JPC. A posteriori time-varying filtering of averaged evoked potentials. I. Introduction and conceptual basis. Biol. Cybern, 1981; 41: $211-222$.

[8] de Weerd JPC and Kap JI. A posteriori time-varying filtering of averaged evoked potentials. II. Mathematical and computational aspects. Biol. Cybern, 1981; 41: 223-234.

[9] Donoho D. De-noising by soft-thresholding. IEEE Transl.Inf. Theory, 1995; 41: 613-627. 
[10] Doyle DJ. Some comments on the use of Wiener filtering for the estimation of evoked potentials. Electr. Clin. Neurophysiol. 1975; 38: 533-534.

[11] Grossmann and Morlet. SIAM J. Math. Anal., 1984; 15: 723-736.

[12] Lopes da Silva F H. Event-related potentials: Methodology and quantification. In: E. Niedermeyer and F. H. Lopes da Silva (Eds.), Electroencephalography: Basic Principles, Clinical Applications and Related Fields. Baltimore: Williams and Wilkins 3rd ed., 1993a, pp: 1097-1123.

[13] Mallat S. A theory for multiresolution signal decomposition: the wavelet representation. IEEE Trans. Pattern Analysis and Machine Intell., 1989; $2(7): 674-693$.

[14] McGillem CD and Aunon JL. Measurements of signal components in single visually evoked brain potentials. IEEE Transl. Biomed. Eng. 1977; 24: $232-241$.

[15] Quian Quiroga R, Başar E and Schürmann M. Phase locking of eventrelated alpha oscillations. In: Chaos in brain? K Lehnertz, CE Elger, J Arnhold and P Grassberger (eds). World Scientific, 2000. pp:301-304.

[16] Quian Quiroga R and Schürmann M. Clin. Neurophysiol., 1999; 110: 643-655.

[17] Regan D. Human brain electrophysiology. Evoked potentials and evoked magnetic fields in science and medicine. Elsevier, Amsterdam, 1989.

[18] Schiff S.J., Aldrouby A, Unser M and Sato S. Fast wavelet transformation of EEG. Electr. Clin. Neurophysiol., 1994a, 91: 442-455.

[19] Walter DO. A posteriori "Wiener filtering" of average evoked responses. Electr. Clin. Neurophysiol. (Suppl.), 1969; 27: 61-70. 
[20] Woody CD. Characterization of an adaptive filter for the analysis of variable latency neuroelectric signals. Med. Biol. Eng, 1967; 5: 539-553.

[21] Unser M, Aldroubi A and Eden M. On the asymptotic convergence of B-Spline wavelets to Gabor functions. IEEE Trans. Inf. Theory, 1992; 38: 864-872. 


\begin{tabular}{ccccc}
$\begin{array}{c}\text { Subject } \\
\text { (age) }\end{array}$ & $\begin{array}{c}\text { Ampl. N100 } \\
\text { (S.D.) }\end{array}$ & $\begin{array}{c}\text { latency N100 } \\
\text { (S.D.) }\end{array}$ & cross-corr. & selected trials \\
\hline $427(45)$ & $3.80(4.24)$ & $127(2.69)$ & 0.43 & $28 / 50(46 \%)$ \\
$594(37)$ & $5.09(5.46)$ & $117(2.42)$ & 0.40 & $25 / 40(52 \%)$ \\
$492(70)$ & $3.68(9.52)$ & $132(3.61)$ & 0.40 & $16 / 50(32 \%)$ \\
$1558(74)$ & $1.87(8.95)$ & $107(3.13)$ & 0.30 & $16 / 40(35 \%)$ \\
EEG & $1.95(12.00)$ & $93(5.93)$ & 0.14 & $6 / 30(13 \%)$
\end{tabular}

Table 1: Results of the quantitative analysis of auditory evoked potentials (N100). Note the higher variability in the amplitude and latency of the N100 in the older subjects. In the last row we include the values for a control EEG signal (without evoked potentials). 


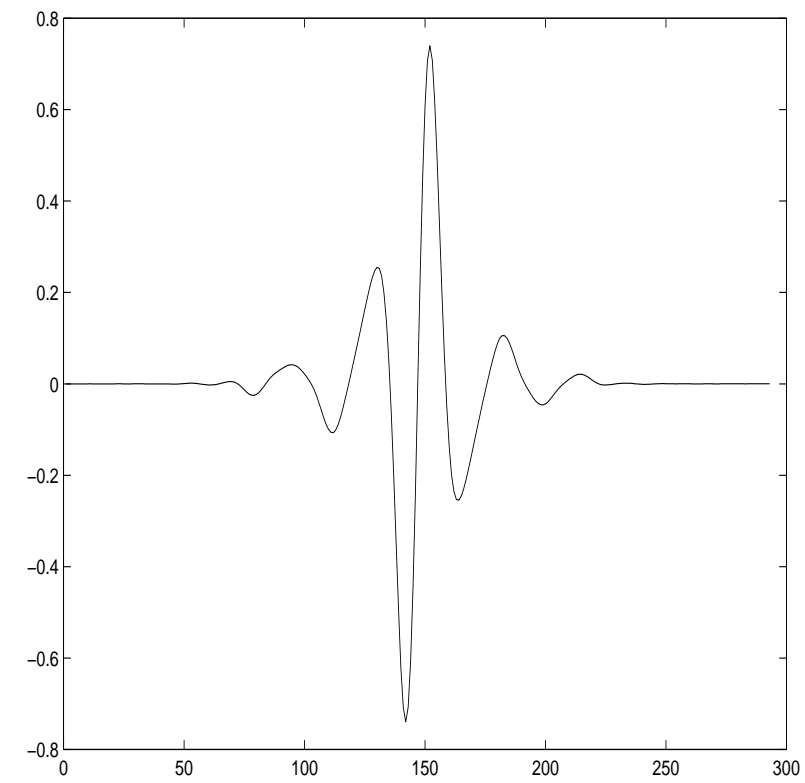

Figure 1: Quadratic B-Spline wavelet function. 

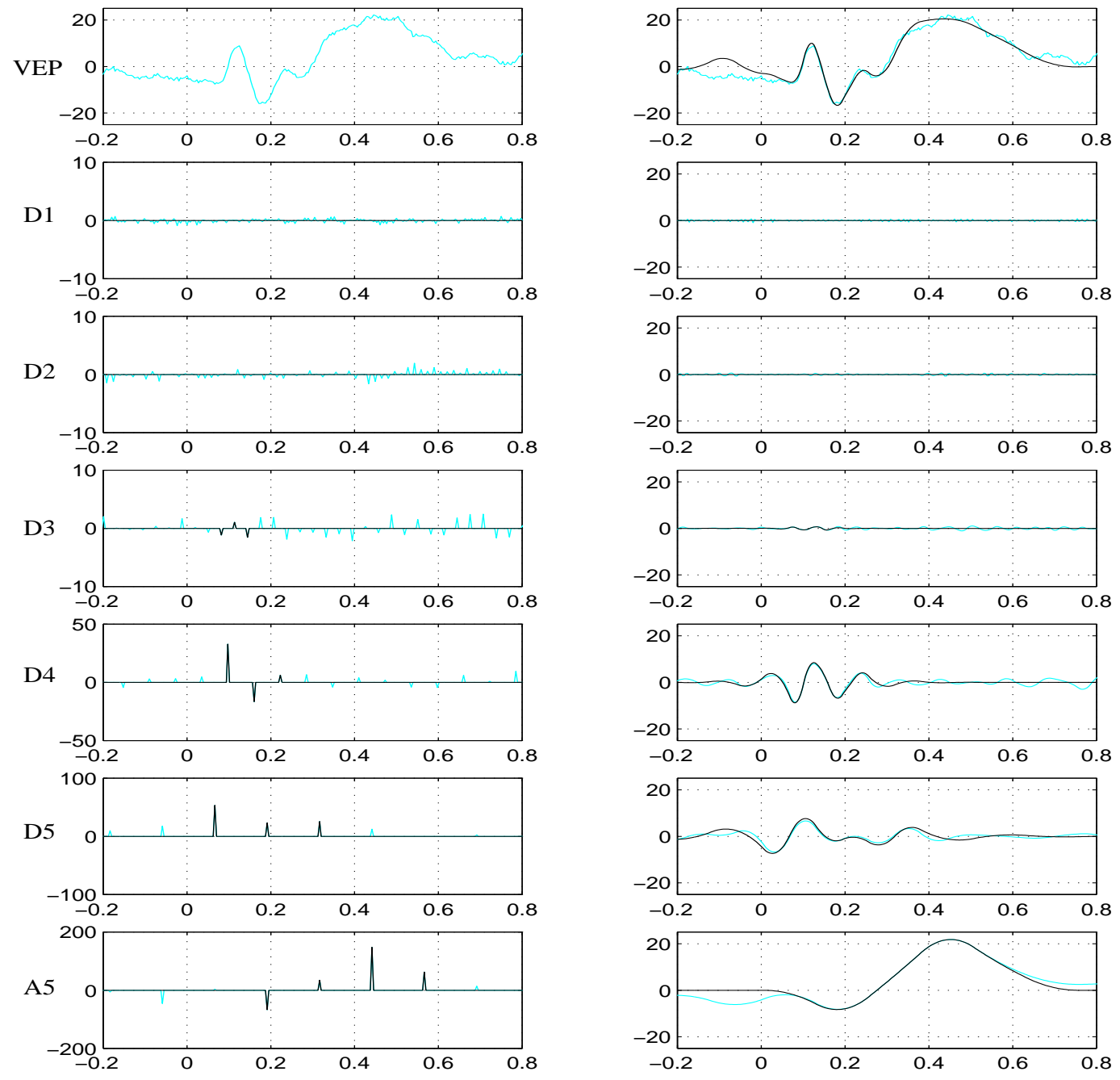

TIME

Figure 2: Multiresolution decomposition and reconstruction of an averaged visual evoked potential (target stimuli). Gray curves: original decomposition and reconstruction; black curves: denoised decomposition and reconstruction. $d_{1}-d_{5}$ are the details at different scales and $a_{5}$ is the last approximation. Note how the denoised reconstruction filters the noisy activity contaminating the original average. 

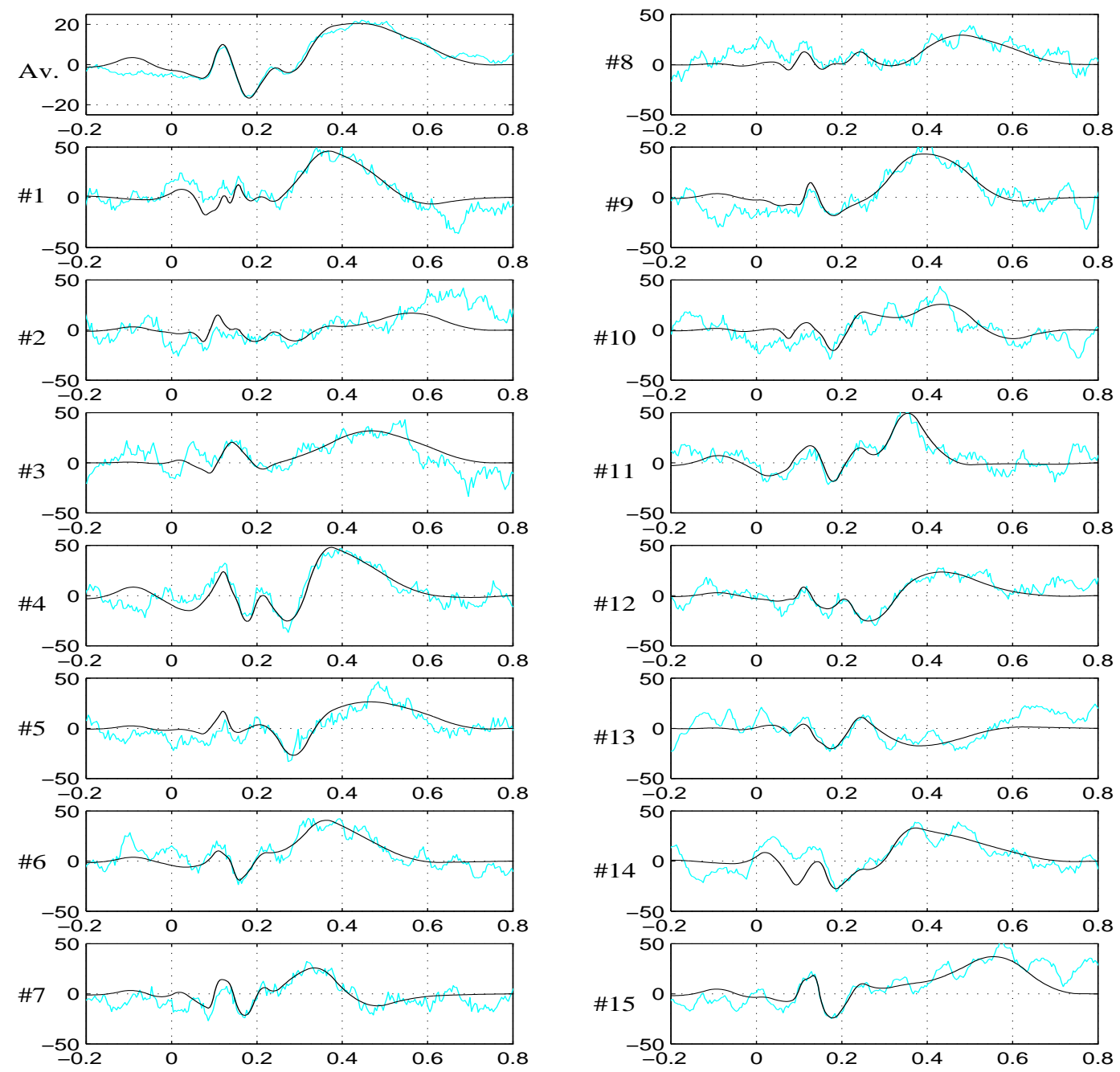

TIME

Figure 3: Single trials corresponding to the average of the previous figure. Grey lines: original data; black lines: denoised data. Note how after denoising the evoked potentials (as seen in the average) are recognizable in most of the single trials from the background EEG. 
Figure 4: [COLOR] Contour plot of the single trials shown in the previous figure. After denoising (rigth plot) it is possible to see the evolution of the evoked responses with the trial number. This is hardly recognizable from the original signal (left plot).

Figure 5: [COLOR] Contour plot of an ongoing electroencephalogram (without evoked potentials) and its denoising. Note that in this case no coherent structure is visible in the denoised signal.

Figure 6: [COLOR] auditory evoked potential "target trials" of a typical subject (subject:427, age: 45) and the denoised responses. Note in particular the decrease of the P300 response after the fifth trial. Again, these patterns are hardly recognizable from the original signal.

Figure 7: [COLOR] auditory evoked potentials "non-target trials" of the subject of the previous figure. As expected, no pattern related with a P300 is seen. 
Figure 8: Averaged auditory evoked potentials, selective averages and jitter corrected averages for the 4 subjects studied and the spontaneous EEG signal. Note how selective and jitter corrected averages increase the definition of the evoked potentials (except for subject 1558 where changes are comparable to the ones obtained in the control EEG signal. 
This figure "figure4.jpg" is available in "jpg" format from: http://arxiv.org/ps/nlin/0006027v1 
This figure "figure5.jpg" is available in "jpg" format from: http://arxiv.org/ps/nlin/0006027v1 
This figure "figure6.jpg" is available in "jpg" format from: http://arxiv.org/ps/nlin/0006027v1 
This figure "figure7.jpg" is available in "jpg" format from: http://arxiv.org/ps/nlin/0006027v1 
This figure "figure8.jpg" is available in "jpg" format from: http://arxiv.org/ps/nlin/0006027v1 